\title{
POLYNOMIAL APPROXIMATION IN THE MEAN WITH RESPECT TO HARMONIC MEASURE ON CRESCENTS
}

\author{
JOHN AKEROYD
}

\begin{abstract}
For $1 \leq s<\infty$ and "nice" crescents $G$, this paper gives a necessary condition (Theorem 2.6) and a sufficient condition (Theorem 2.5) for density of the polynomials in the generalized Hardy space $H^{s}(G)$. These conditions are easily tested and almost equivalent.
\end{abstract}

The problem of polynomial approximation in the mean with respect to area measure on crescents has been extensively researched by such authors as J. Brennan [1] and S. Mergelyan [6]. This author explores the same problem except that area measure is replaced by harmonic measure. Hence, the question here may be rephrased: given $1 \leq s<\infty$, for which crescents $G$ are the polynomials dense in the generalized Hardy space $H^{s}(G)$ (the collection of all analytic functions $f$ on $G$ for which $|f|^{s}$ has a harmonic majorant on $G$. For $z_{0}$ in $G,\|f\|_{z_{0}}=u_{f}\left(z_{0}\right)^{1 / s}$, where $u_{f}$ is the least harmonic majorant of $|f|^{s}$ on $\left.G\right)$ ?

1. Preliminaries. Suppose $G$ is a bounded Dirichlet region in the complex plane (that is, $G$ is a bounded, open, connected set on which the Dirichlet problem is solvable). If $z \in G$, then define

$$
\rho_{z}: C_{\mathbf{R}}(\partial G) \rightarrow \mathbf{R} \quad \text { by } \quad \rho_{z}(f)=\hat{f}(z),
$$

where $\hat{f}$ is the solution to the Dirichlet problem on $G$ with boundary values $f$. By the maximum principle, $\rho_{z}$ is a bounded, positive linear functional on $C_{\mathbf{R}}(\partial G)$. Since $\partial G$ is compact, the Riesz representation theorem gives a unique Borel measure $\omega \equiv \omega(\cdot, G, z)$ (indeed a probability measure by the maximum principle and the fact that $\left.\rho_{z}(1)=1\right)$ with $\operatorname{support}(\omega) \subseteq \partial G$ such that $\rho_{z}(f)=\int f d \omega$ for all $f$ in $C_{\mathbf{R}}(\partial G)$. This measure is called harmonic measure for $G$ at $z$. To familiarize oneself with some of the properties of harmonic measure one may consult [4]. Throughout this paper, " $m$ " denotes Lebesgue measure on $\mathbf{R}$.

1.1 Definition. A crescent is a region (in the complex plane $\mathbf{C}$ ) bounded by two Jordan curves which intersect in a single point such that one of the Jordan curves is internal to the other.

If $G$ is a crescent, then denote the outer boundary of $G$ by $\partial_{\infty} G \equiv \partial \bar{G}^{\wedge}\left(\bar{G}^{\wedge}\right.$ being the polynomially convex hull of the closure of $G$ ) and the inner boundary

Received by the editors September 4, 1986. This paper was presented at the International Conference on Harmonic Measure and Potential Theory in Euclidean Spaces (7/30/86), sponsored by The University of Toledo. Related topics were presented at the AMS meeting (1/21/87).

1980 Mathematics Subject Classification (1985 Revision). Primary 30E10; Secondary 30C85, 30D55, 30H05, 47A10, 47B20.

This paper is derived from part of the author's Ph.D. dissertation written at Indiana University under the direction of John B. Conway. Part of this research was done while the author received support from the National Science Foundation grant MCS 83-204-26. 
of $G$ by $\overline{\partial_{0} G}$, where $\partial_{0} G \equiv\{\partial G\} \backslash\left\{\partial_{\infty} G\right\}$. The multiple boundary point of $G$ is defined to be $\left\{\partial_{\infty} G\right\} \cap\left\{\overline{\partial_{0} G}\right\}$. The reader should note that crescents are simply connected Dirichlet regions.

The following two propositions are primarily due to the relationship between conformal maps and harmonic measure. The proof of each is left as an exercise.

1.2 Proposition. Suppose that $G$ is a crescent and $\omega$ is harmonic measure for $G$ at $z_{0}$ in $G$. Let $E$ be a subregion of $G$ such that $\partial E$ is a Jordan curve and $\Gamma \equiv\{\partial E\} \cap\{\partial G\}$ is a Jordan arc with endpoints $\alpha$ and $\beta$. Let $\nu$ be harmonic measure for $E$ at $\xi_{0}$ in $E$. If $K$ is a compact subset of $\Gamma \backslash\{\alpha, \beta\}$, then there exists a constant $r \equiv r(K)>0$ such that $\left.(1 / r) \cdot \nu\right|_{K} \leq\left.\omega\right|_{K} \leq\left. r \cdot \nu\right|_{K}$.

1.3 Proposition. Suppose that $G$ is a crescent with multiple boundary point $\lambda$, and $\omega$ is harmonic measure for $G$ at $z_{0}$ in $G$. If $\nu$ is harmonic measure for $\operatorname{int}\left(\bar{G}^{\wedge}\right)$ at $z_{0}$, then, for any compact subset $K$ of $\left\{\partial_{\infty} G\right\} \backslash\{\lambda\}$, there exists a constant $r \equiv$ $r(K)>0$ such that $\left.\omega\right|_{K} \leq\left.\nu\right|_{K} \leq\left. r \cdot \omega\right|_{K}$. Consequently, $\int_{K} \log (d \omega / d \nu) d \nu>-\infty$.

If $K$ is a compact subset of $\mathbf{C}, \sigma$ is a regular Borel measure with support in $K$ and $1 \leq s<\infty$, then $P^{s}(\sigma)$ [resp. $R^{s}(K, \sigma)$ ] denotes the closure of the polynomials [resp. the rational functions with the poles off $K$; $\operatorname{Rat}(K)]$ in $L^{s}(\sigma)$. If $G$ is a crescent, $\omega$ is harmonic measure for $G$ at $z_{0}$ in $G$ and $1 \leq s<\infty$, then one can show that $R^{s}(\bar{G}, \omega)$ is isometrically isomorphic to $H^{s}(G)$ (the collection of all analytic functions $f$ on $G$ such that $|f|^{s}$ has a harmonic majorant on $G \cdots\|f\|_{z_{0}}=u_{f}\left(z_{0}\right)^{1 / s} ; u_{f}$ is the least harmonic majorant of $|f|^{s}$ on $G$ ).

2. For which crescents $G$ is $P^{s}\left(\omega\left(\cdot, G, z_{0}\right)\right)=H^{s}(G)$ ? The primary focus of this paper is the following question, which is a paraphrase of the above title.

2.1 Question. If $1 \leq s<\infty$, then for which crescents $G$ is $P^{s}(\omega)=H^{s}(G)$, where $\omega$ is harmonic measure for $G$ at some $z_{0}$ in $G$ ?

The following proposition (whose proof is left as an excercise) encourages one to believe that there is an answer to Question 2.1 which solely involves "geometric" conditions.

2.2 Proposition. Suppose $1 \leq s<\infty, G$ is a crescent, $\omega$ is harmonic measure for $G$ at $z_{0}$ in $G$ and $P^{s}(\omega)=H^{s}(G)$. If $E$ is a crescent such that $E \subseteq G$ and $\left\{\bar{G}^{\wedge} \backslash \bar{G}\right\} \subseteq\{\bar{E} \backslash \backslash \bar{E}\}$, and if $\nu$ is harmonic measure for $E$ at $\xi_{0}$ in $E$, then $P^{s}(\nu)=H^{s}(E)$.

Rather than assaulting Question 2.1 in its full generality, this paper focuses on a collection of "nice" crescents.

Let $\mathcal{F}$ be the collection of all functions $f$ which have the following properties:

(i) $f: \mathbf{R} \rightarrow \mathbf{R}, f(x)=f(-x), f \geq 0$ and $f(x)=0$ if and only if $x=0$.

(ii) $f$ is continuously differentiable, $f^{\prime}$ is nondecreasing and $f^{\prime}(0)=0$.

(iii) There exist constants (depending on $f$ ) $\beta>\alpha>1$ and $\delta>0$ such that $x^{\beta} \leq f(x) \leq x^{\alpha}$ whenever $0<x<\delta$.

The assumptions that $f$ is an even function and that $f^{\prime}$ is continuous are made for convenience of proof; they can be eliminated. Furthermore, Proposition 2.2 later reveals that, for our purposes, condition (iii) is scarcely a restriction.

If $t>0$ and $f \in \mathcal{F}$, then let $A(t)=\{z \in \mathbf{C}: \operatorname{Im}(z)>0$ and $|z|<t\}$ and $B(f, t)=\{z=x+i y: y \geq f(x)$ and $|z| \leq t\}$. Let $C=\{A(R) \backslash B(f, r): 0<r<R$ 
and $f \in \mathcal{F}\}$. C is seen to be a collection of crescents, each crescent of which lies in the upper half-plane, is symmetric about the imaginary axis and has $z=0$ as its multiple boundary point. Moreover, $C$ is substantial in the sense that a wide variety of rates of "thinning", near the multiple boundary point, are represented by crescents in $C$.

If $f \in \mathcal{F}, 0<\theta, \tau<\pi / 2$ and $n$ is any positive integer, then let $S(\tau)=\left\{t e^{i \tau}: t>\right.$ $0\}$ and

$$
\xi_{n} \equiv \xi_{n}(\theta, f)=\left\{\begin{array}{l}
\{x+i f(x): x \in \mathbf{R}\} \cap S(\theta / n) \quad \text { if nonempty } \\
0 \quad \text { otherwise. }
\end{array}\right.
$$

By definition of $\mathcal{F}, \xi_{n}$ is always a well-defined complex number. Let $x_{n} \equiv x_{n}(\theta, f)=$ $\operatorname{Re}\left(\xi_{n}(\theta, f)\right)$ and $\rho_{n} \equiv \rho_{n}(\theta, f)=\left|\xi_{n}(\theta, f)\right|$. If $\xi_{n} \neq 0$, then $\xi_{k} \neq 0$ for all $k \geq n$ and

$$
\rho_{n}>\rho_{n+1}>\cdots>\rho_{n+j} \rightarrow 0 \quad \text { as } j \rightarrow \infty \text {. }
$$

2.3 LEMMA. If $f \in \mathcal{F}$ and $\int_{0}^{\varepsilon}\left(f^{\prime}\right)^{-1} d m=\infty$ for some $\varepsilon>0$, then $\sum_{n=1}^{\infty} \rho_{n}(\theta, f)$ $=\infty$ whenever $0<\theta<\pi / 2$.

ProOF. Suppose that $\sum_{n=1}^{\infty} \rho_{n}(\theta, f)<\infty$ for some $\theta, 0<\theta<\pi / 2$. Hence $\sum_{n=1}^{\infty} x_{n}<\infty\left(x_{n} \equiv x_{n}(\theta, f)\right)$. Now, there exists $N \geq 1$ such that for all $n \geq$ $N, x_{n}>0$. For $n \geq N$, let $t_{n} \equiv t_{n}(\theta, f)=\min \left\{x: f^{\prime}(x)=\theta / n\right\}$. Since $f^{\prime}$ is nondecreasing and $f^{\prime}(0)=f(0)=0$, it follows that (for $n \geq N$ ) $f^{\prime}\left(x_{n}\right)>\theta / n$, and so $t_{n}<x_{n}$. Therefore,

$$
\sum_{n=N}^{\infty} t_{n}\left(f^{\prime}\left(t_{n+1}\right)^{-1}-f^{\prime}\left(t_{n}\right)^{-1}\right)=\frac{1}{\theta} \cdot \sum_{n=N}^{\infty} t_{n}((n+1)-n)=\frac{1}{\theta} \cdot \sum_{n=N}^{\infty} t_{n}<\infty .
$$

Since $\left.\left(f^{\prime}\right)^{-1}\right|_{(0, \infty)}$ is nonincreasing, we can conclude that

$$
\int_{0}^{\varepsilon}\left(f^{\prime}\right)^{-1} d m<\infty \text { Q.E.D. }
$$

2.4 LEMMA. Suppose that $f \in \mathcal{F}$ and $\omega$ is harmonic measure for $G \equiv$ $A(R) \backslash B(f, r) \in \mathcal{C}$ at $z_{0}$ in $G$. If $1 \leq s<\infty$ and $\sum_{n=1}^{\infty} \rho_{n}(\theta, f)=\infty$ whenever $0<\theta<\pi / 2$, then $\sum_{n=1}^{\infty}\left\|z^{-2 n}\right\|_{s}^{-1 / 2 n}=\infty\left(z^{-n} \in L^{s}(\omega)\right.$ for $\left.n=1,2,3, \ldots\right)$.

ProOF. We may assume that $s$ is a positive integer. Choose $\theta=\pi / 8 s$. Let $z=t e^{i \varphi}$ and $h_{n, s}(z)=\operatorname{Re}\left(z^{-2 n s}\right)=t^{-2 n s} \cdot \cos (2 n s \varphi)$. Now there exists a positive integer $N$ such that $0<\rho_{n}(\theta, f)<r$ whenever $n \geq N$. Hence, for $n \geq N$ and $\rho_{n} \equiv \rho_{n}(\theta, f)(\theta=\pi / 8 s)$

$$
\begin{aligned}
\left\|z^{-2 n}\right\|_{s}^{s} & =\int\left|z^{-2 n}\right|^{s} d \omega=\int_{B\left(0 ; \rho_{n}\right)}\left|z^{-2 n}\right|^{s} d \omega+\int_{\bar{G} \backslash B\left(0 ; \rho_{n}\right)}\left|z^{-2 n}\right|^{s} d \omega \\
& \leq 2 \cdot \int_{B\left(0 ; \rho_{n}\right)} h_{n, s} d \omega+\int_{\bar{G} \backslash B\left(0 ; \rho_{n}\right)}\left|z^{-2 n}\right|^{s} d \omega \\
& \left.\quad \text { since } h_{n, s}(z) \geq\left|z^{-2 n}\right|^{s} / 2 \text { for } z \text { in } B\left(0 ; \rho_{n}\right) \cap \bar{G}\right) \\
& \leq 2 \cdot \int h_{n, s} d \omega+3 \cdot \int_{\bar{G} \backslash B\left(0 ; \rho_{n}\right)}\left|z^{-2 n}\right|^{s} d \omega \\
& \leq 2\left|z_{0}\right|^{-2 n s}+3 \rho_{n}^{-2 n s} \quad\left(\text { since } \int h_{n, s} d \omega=h_{n, s}\left(z_{0}\right)\right) \\
& \leq 3 C^{-2 n s} \cdot \rho_{n}^{-2 n s},
\end{aligned}
$$


where $C=\left(\left|z_{0}\right|^{-1}+1\right)^{-1}$. Therefore,

$$
\left\|z^{-2 n}\right\|_{s}^{-1 / 2 n} \geq C 3^{-1 / 2 n s} \cdot \rho_{n} \geq(C / 2) \cdot \rho_{n} .
$$

Thus,

$$
\sum_{n=1}^{\infty}\left\|z^{-2 n}\right\|_{s}^{-1 / 2 n} \geq \sum_{n=N}^{\infty}\left\|z^{-2 n}\right\|_{s}^{-1 / 2 n} \geq \frac{C}{2} \cdot \sum_{n=N}^{\infty} \rho_{n}=\infty
$$

The following theorem is a relative of [3, Theorem 2], where the context is polynomial approximation in the mean for area measure on crescents.

2.5 THEOREM. Suppose that $f \in \mathcal{F}, \omega$ is harmonic measure for $G \equiv$ $A(R) \backslash B(f, r) \in C$ at $z_{0}$ in $G$ and $1 \leq s<\infty$. If $\int_{0}^{\varepsilon}\left(f^{\prime}\right)^{-1} d m=\infty$ for some $\varepsilon>0$, then $P^{s}(\omega)=H^{s}(G)$.

Proof. To prove the theorem it is sufficient to show that if $g \in L^{s}(\omega)^{*}=$ $L^{t}(\omega)(1 / s+1 / t=1)$ and $g \perp P^{s}(\omega)$, then $g \perp H^{s}(G)\left(\cong R^{s}(\bar{G}, \omega)\right)$. That is, if $g \in L^{t}(\omega), \hat{g}(\xi) \equiv \int g(z) /(z-\xi) d \omega(z)$ and $\left.\hat{g}\right|_{C \backslash\left\{\bar{G}^{\wedge}\right\} \equiv} 0$, then $\left.\hat{g}\right|_{\left\{\bar{G}^{\wedge}\right\} \backslash \bar{G}} \equiv 0$. In light of this, choose $g$ in $L^{t}(\omega)$ such that $\left.\hat{g}\right|_{\mathbf{C} \backslash\left\{\bar{G}^{\wedge}\right\}} \equiv 0$. Since $f \in \mathcal{F}$, there exists $\alpha>1$ and $\delta, 0<\delta<r /(1+r)$, such that $f(x) \leq x^{\alpha}$ whenever $0<x<\delta$ (we may assume that $1<\alpha<2)$. Let $\Omega=\left\{z=x+i y: y>|x|^{(\alpha+1) / 2}\right.$ and $\left.|z|<\delta\right\}$.

There exists $k>0$ such that for all $z$ in $\bar{G}$ and $\xi$ in $\bar{\Omega}$,

$$
|z-\xi| \geq k|z|^{2} \text {. }
$$

Moreover, for $z$ in $\bar{G} \backslash\{0\}, \xi$ in $\bar{\Omega}$ and any positive integer $n$,

$$
\frac{1}{z-\xi}=\frac{1}{z}+\cdots+\frac{\xi^{n-1}}{z^{n}}+\frac{\xi^{n}}{z^{n}(z-\xi)} .
$$

By Lemma 2.4, Runge's theorem, the geometry of $G$ and Lebesgue's dominated convergence theorem, $a_{i}(z) \equiv \xi^{i-1} / z^{i} \in P^{s}(\omega)$ for positive integers $i$. Since $g \perp$ $P^{s}(\omega)$, it follows that

$$
\hat{g}(\xi)=\xi^{n} \cdot \int \frac{g(z)}{z^{n}(z-\xi)} d \omega(z)
$$

Therefore,

$$
\begin{aligned}
|\hat{g}(\xi)| & \leq|\xi|^{n} \cdot \int \frac{|g(z)|}{\left|z^{n}\right||z-\xi|} d \omega(z) \\
& \leq \frac{|\xi|^{n}}{k} \cdot \int\left|g(z) \| z^{-n-2}\right| d \omega(z) \\
& \leq \frac{|\xi|^{n}}{k} \cdot\left\|z^{-n-2}\right\|_{s} \cdot\|g\|_{t} .
\end{aligned}
$$

Let $\varphi: \mathbf{D} \rightarrow \Omega(\mathbf{D}=\{z:|z|<1\})$ be the Riemann map such that $\varphi(1)=0$. Let $\psi(w)=(\hat{g} \circ \varphi)(w)$ and note that

$$
|\psi(w)| \leq|| 1-w)\left.\cdot \frac{\varphi(w)}{1-w}\right|^{n} \cdot \frac{\|g\|_{t}}{k} \cdot\left\|z^{-n-2}\right\|_{s}
$$

By [7, Theorem ix. 9. (ii)],

$$
0<\sup _{w \in \mathbf{D}}\left|\frac{\varphi(w)}{1-w}\right|=M<\infty
$$


Therefore,

$$
|\psi(w)| \leq|1-w|^{n} \cdot M^{n} \cdot\left\|z^{-n-2}\right\|_{s} \cdot\|g\|_{t} / k
$$

We may assume that $\|g\|_{t} \leq k /\left\|z^{-2}\right\|_{s}$ and that $|\psi(w)| \leq 1$ for all $w$ in $\mathbf{D}$. Hence,

$$
|\psi(w)| \leq|1-w|^{n} \cdot M^{n} \cdot\left\|z^{-n-2}\right\|_{s} /\left\|z^{-2}\right\|_{s}
$$

for $n=0,1,2, \ldots$ Let $A_{n}=M^{n} \cdot\left\|z^{-n-2}\right\|_{s} /\left\|z^{-2}\right\|_{s}$. An easy exercise (left to the reader) shows that $\left\{A_{n}\right\}_{n=0}^{\infty}$ is a log-convex sequence. In other words $A_{0}=1$ and, for $n \geq 1, A_{n}^{2} \leq A_{n-1} \cdot A_{n+1}$. Hence, for $n \geq 1$,

$$
A_{n}^{1 / n} \leq A_{n+1}^{1 /(n+1)}
$$

Now

$$
A_{n}^{-1 / n} \geq \frac{1}{M} \cdot\left[\frac{\left\|z^{-2}\right\|_{s} \cdot\left\|z^{-4}\right\|_{s}}{\left\|z^{-2 n}\right\|_{s}}\right]^{1 / 2 n}
$$

and so, from the hypothesis, Lemmas 2.3 and 2.4 , it follows that $\sum_{n=1}^{\infty} A_{n}^{-1 / n}=\infty$. With this fact and (2.5.2), [2, p. 50] tells us that $\psi \equiv 0$. Therefore, $\left.\hat{g}\right|_{\Omega} \equiv 0$. Yet $\hat{g}$ is analytic off the support of $\omega$. So $\left.\hat{g}\right|_{\left\{\bar{G}^{\wedge}\right\} \backslash \bar{G}} \equiv 0$ and the result follows.

2.6 THEOREM. Suppose that $f \in \mathcal{F}, \omega$ is harmonic measure for $G \equiv$ $A(R) \backslash B(f, r) \in C$ at $z_{0}$ in $G$ and $1 \leq s<\infty$. If $P^{s}(\omega)=H^{s}(G)$, then

$$
\int_{0}^{\varepsilon}\left(f^{\prime}(x)\right)^{-1} \cdot|\log (x)| d m(x)=\infty \quad \text { for } \varepsilon>0 .
$$

ProOF. Assume that, for some positive $\varepsilon$,

$$
\int_{0}^{\varepsilon}\left(f^{\prime}(x)\right)^{-1} \cdot|\log (x)| d m(x)<\infty
$$

We want to show that $P^{s}(\omega) \neq H^{s}(G)$. One may assume that $r=3$ (exercise). Let $\nu$ be harmonic measure for $\operatorname{int}\left(\bar{G}^{\wedge}\right)$ at $z_{0}$. By [5] Szegö's Theorem 8.2, the symmetry of $G$ and Proposition 1.3, if $\left.\omega_{\infty} \equiv \omega\right|_{\partial_{\infty} G}$ and there exists $\varepsilon, 0<\varepsilon<3$, such that $\int_{0}^{\varepsilon} \log \left(d \omega_{\infty} / d \nu\right) d \nu>-\infty$, then $P^{s}\left(\omega_{\infty}\right) \neq R^{s}\left(\partial_{\infty} G, \omega_{\infty}\right)$ and hence $P^{s}(\omega) \neq$ $H^{s}(G)$. By the nature of $\nu$, this reduces to showing that $\int_{0}^{\varepsilon} \log \left(d \omega_{\infty} / d m\right) d m>$ $-\infty$.

With our strategy established, let us turn our attention to a crescent which simplifies estimates; estimates that can be applied to $G$. Let $E=G \cup\{z: \operatorname{Im}(z) \leq 0$ and either $|z-1|<\frac{1}{4}$ or $\left.|z+1|<\frac{1}{4}\right\}$, and let $\sigma$ be harmonic measure for $E$ at $z_{0}$. If $0<x<\frac{1}{2}$, then let $\theta_{x}=\tan ^{-1}\left(f^{\prime}(x)\right)$ and $l_{x}=\left\{x+i f(x)+r e^{i \theta_{x}}: r \in \mathbf{R}\right\}$. Let $r_{x}=l_{x} \cap \mathbf{R}, d_{x}=r_{x}+e^{i \theta_{x} / 2} \in G$ and $\sigma_{x}$ be harmonic measure for $E$ at $d_{x}$ (see Figure 1).

If $U_{x}=\left\{z \in E: 0<\arg \left(z-r_{x}\right)<\theta_{x}\right.$ and $\left.\left|z-r_{x}\right|<2\right\}$, then let $\eta_{x}$ be harmonic measure for $U_{x}$ at $d_{x}$. Notice that $U_{x}=\left\{z: 0<\arg (z)<\theta_{x}\right.$ and $\left.|z|<2\right\}+r_{x}$. (For $\psi_{x}(z)=\left(z-r_{x}\right)^{\pi / \theta_{x}}$, let $\mu_{x}$ be harmonic measure for $\psi_{x}\left(U_{x}\right)$ at $\psi_{x}\left(d_{x}\right)=i$. Since $[-1,1] \subset \partial\left\{\psi_{x}\left(U_{x}\right)\right\}$ and $\{z: \operatorname{Im}(z)>0$ and $|z|<2\} \subseteq \psi_{x}\left(U_{x}\right)$ whenever $0<x<\frac{1}{2}$, there exists a constant $k>0$ such that,

$$
\left.d \mu_{x} /\left.d m\right|_{[0,1 / 2]}>k, \quad \text { for } 0<x<1 / 2 .\right)
$$

Now $d_{x} \rightarrow 1 \in E$ as $x$ decreases to zero and so [4] Proposition 7.13 provides us with a constant $A>0$ such that

$$
d \sigma / d \sigma_{x} \geq A,
$$




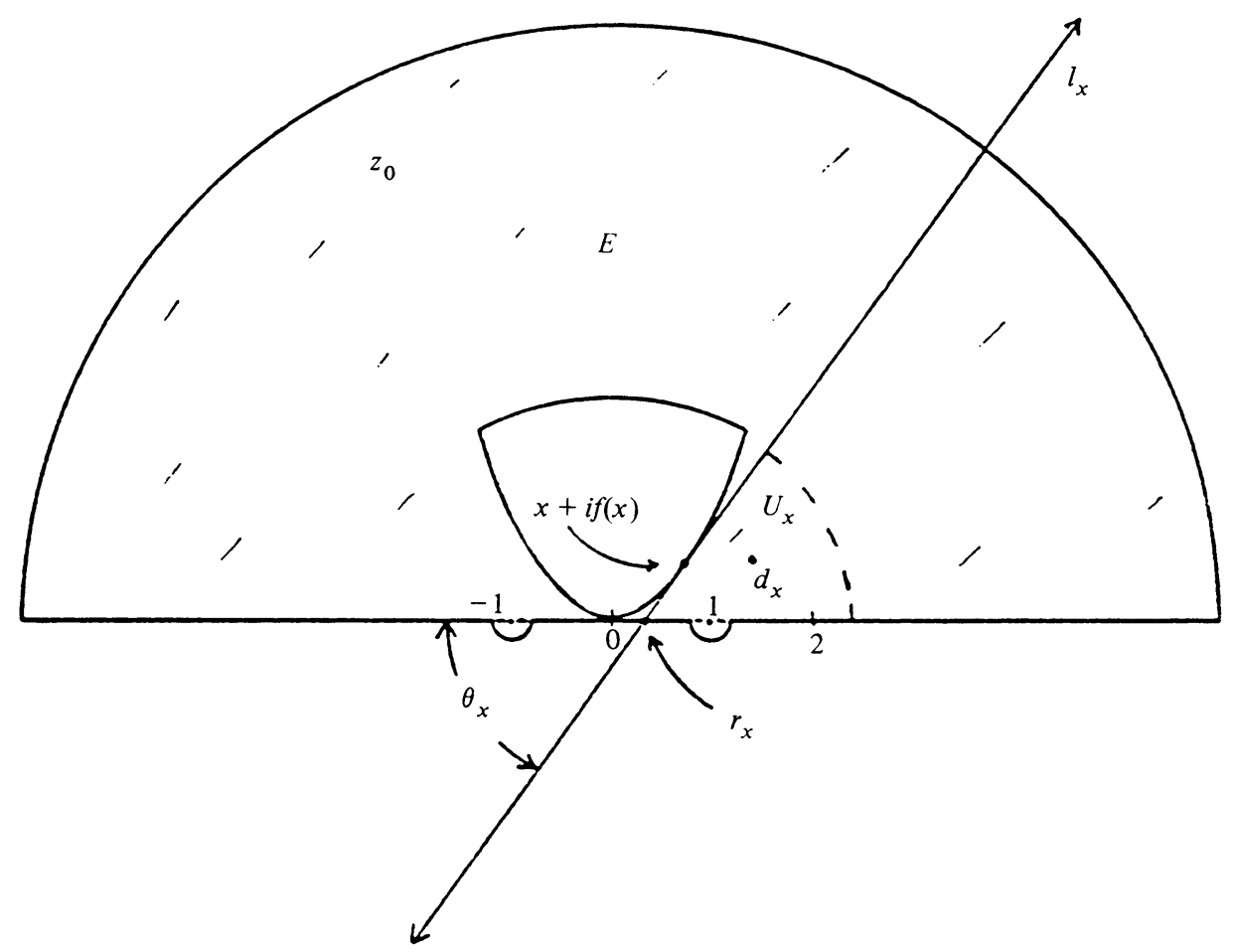

FIGURE 1

whenever $0<x<\frac{1}{2}$. Since $U_{x} \subseteq E$,

$$
d \sigma_{x}(z) / d \eta_{x} \geq 1
$$

for $0<x<\frac{1}{2}$ and $r_{x} \leq z<\frac{1}{2}$. Furthermore, by standard estimates, if $0<x<\frac{1}{2}$ and $r_{x}<z<\frac{1}{2}$, then

$$
\frac{d \eta_{x}}{d m}(z) \geq \frac{k \pi}{\theta_{x}}\left(z-r_{x}\right)^{\left(\pi / \theta_{x}\right)-1} \geq 2 k\left(z-r_{x}\right)^{\pi / \theta_{x}}
$$

where $k$ is as in (2.6.1).

Note that if $0<x<y<\frac{1}{2}$, then $0<r_{x} \leq r_{y}<\frac{1}{2}$. Moreover, $r_{x} \rightarrow 0$ as $x$ decreases to zero. If $0<x<\frac{1}{2}$, then $x \in\left(r_{x}, \frac{1}{2}\right), \theta_{x} \leq \theta_{1 / 2}<\pi / 2$ and $f^{\prime}(x) / \theta_{x}=\left(\tan \theta_{x}\right) / \theta_{x} \rightarrow 1$ as $x$ decreases to zero. So, there exists a constant $a>0$ such that if $0<x<\frac{1}{2}$, then $\theta_{x} \geq a \cdot f^{\prime}(x)$ and thus, since $r_{x}<x<\frac{1}{2}$,

$$
d \eta_{x}(x) / d m>2 k\left(x-r_{x}\right)^{\pi / \theta_{x}} \geq 2 k\left(x-r_{x}\right)^{(\pi / a) \cdot\left(f^{\prime}(x)\right)^{-1}} .
$$

Since $f(x) /\left(x-r_{x}\right) \rightarrow 0$ as $x$ decreases to zero, there exists (by condition (iii) of f) a constant $b>0$ such that $x-r_{x} \geq x^{b}$ for $0<x<\frac{1}{2}$. Therefore,

$$
d \eta_{x}(x) / d m \geq 2 k(x)^{b \pi\left(f^{\prime}(x)\right)^{-1} / a}
$$

whenever $0<x<\frac{1}{2}$.

Combining (2.6.2)-(2.6.5), there exists a constant $B>0$ such that if $0<x<\frac{1}{2}$, then

$$
d \sigma(x) / d m \geq B(x)^{b \pi\left(f^{\prime}(x)\right)^{-1 / a}} .
$$


By Proposition 1.2, there exists a constant $C>0$ such that

$$
d \omega_{\infty}(x) / d m=d \omega(x) / d m \geq C(x)^{b \pi\left(f^{\prime}(x)\right)^{-1} / a}
$$

whenever $0<x<\frac{1}{2}$. Our initial assumption now tells us that

$$
\int_{0}^{\varepsilon} \log \left(d \omega_{\infty} / d m\right) d m>-\infty
$$

and so the conclusion follows.

\section{BIBLIOGRAPHY}

1. J. Brennan, Approximation in the mean by polynomials on non-Carathéodory domains, Ark. Mat. 15 (1977), 117-168.

2. T. Carleman, Fonctions quasi analytiques, Gauthier-Villars, Paris, 1926.

3. J. A. Cima and A. Matheson, Approximation in the mean by polynomials, Rocky Mountain J. Math. 15 (1985), 729-738.

4. J. B. Conway, Subnormal operators, Pitman, 1981.

5. T. W. Gamelin, Uniform algebras, 2nd ed., Chelsea, New York, 1984.

6. S. N. Mergelyan, On the completeness of systems of analytic functions, Amer. Math. Soc. Transl. (2) 19 (1962), 109-166.

7. M. Tsuji, Potential theory in modern function theory, 2nd ed., Chelsea, New York, 1975.

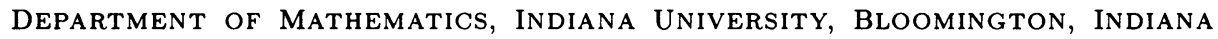
47405

Current address: Department of Mathematics, University of Arkansas, Fayetteville, Arkansas 72701 